\title{
Regression with spatially misaligned data
}

\author{
L. Madsen ${ }^{1 *, \dagger}$, D. Ruppert ${ }^{2}$ and N. S. Altman ${ }^{3}$ \\ ${ }^{1}$ Department of Statistics, Oregon State University, Corvallis, OR 97331, U.S.A. \\ ${ }^{2}$ School of OR and IE, Cornell University, Ithaca, NY, U.S.A. \\ ${ }^{3}$ Department of Statistics, Penn State University, University Park, PA, U.S.A.
}

\begin{abstract}
SUMMARY
We present a simple approach to the problem of estimating the regression slope parameter from spatially misaligned point data. We assume a linear regression model with errors and covariates from two independent Gaussian spatial processes where covariate and response are observed at different locations. Correlation in the covariate is exploited to predict unobserved covariates via kriging. Kriged values are used to find weighted least squares estimates of regression parameters in a 'krige-and-regress' (KR) procedure. The variance of this estimator is calculated, and a variance estimator is proposed. Because the model and assumptions make it possible to write down the joint likelihood of the data, a maximum likelihood (ML) estimator can be found. Under regularity conditions, this estimator is asymptotically normal with asymptotic variance given by the inverse information matrix, which yields a variance estimator for the ML estimator of the regression parameters. The KR and ML estimators are compared in an example using Environmental Protection Agency data and a simulation study is conducted. While the ML estimator of the slope parameter has a smaller variance than the KR estimator, the ML variance estimator is too small to be used for inference whereas the KR variance estimator gives approximately correct inference. Copyright @ 2007 John Wiley \& Sons, Ltd.
\end{abstract}

KEY WORDS: maximum likelihood; kriging

\section{INTRODUCTION}

Advances in data-gathering techniques have led to a wealth of potential predictor variables to explain a spatial response. However, when data come from different sources, the spatial locations and scales rarely coincide. Recent works in reconciling data of incompatible spatial scales are reviewed in Gotway and Young (2002). Much of focus in spatial problems has typically been in spatial prediction or 'kriging'; however, Zhu et al. (2003) tackle a complicated spatial regression problem with misaligned data. In this paper, we present a simpler approach to the problem of estimating the regression slope parameter from spatially misaligned point data when most of the assumptions of simple linear regression are met.

${ }^{*}$ Correspondence to: L. Madsen, Department of Statistics, Oregon State University, Corvallis, OR 97331, U.S.A.

${ }^{\dagger}$ E-mail: madsenl@onid.orst.edu 


\subsection{The model}

Suppose $\mathbf{Y}=\left[Y_{1} \ldots Y_{n}\right]$ is a vector of responses, observed at spatial locations $s_{1} \ldots s_{n}$, and that $X(\cdot)$ and $\epsilon(\cdot)$ are spatially autocorrelated stationary Gaussian random processes defined on a set containing all the $s_{i}$. Let $X_{i}$ and $\epsilon_{i}$ denote $X\left(s_{i}\right)$ and $\epsilon\left(s_{i}\right)$, respectively. Assume that at each location $s_{i}$

$$
Y_{i}=\beta_{0}+\beta_{1} X_{i}+\epsilon_{i}
$$

Let $\mathbf{X}=\left[X_{1} \ldots X_{n}\right]^{\prime}$ and $\boldsymbol{\epsilon}=\left[\epsilon_{1} \ldots \epsilon_{n}\right]^{\prime}$. Assume the predictor process $X(\cdot)$ and the error process $\epsilon(\cdot)$ are independent of each other. Let $\mu_{X}$ denote the mean of the predictor process, and suppose the error process has mean 0 . Suppose further that $\mathbf{X}$ has not been observed, but that observations $\mathbf{W}=\left[W_{1} \ldots W_{m}\right]$ from the predictor process have been observed at spatial locations $t_{1} \ldots t_{m}$, i.e. $W_{i}=X\left(t_{i}\right)$.

The model and assumptions above imply that $\mathbf{Y}, \mathbf{X}$ and $\mathbf{W}$ are jointly normally distributed with mean

$$
E\left[\begin{array}{l}
\mathbf{Y} \\
\mathbf{X} \\
\mathbf{W}
\end{array}\right]=\left[\begin{array}{c}
\left(\beta_{0}+\beta_{1} \mu_{X}\right) \mathbf{1}_{n \times 1} \\
\mu_{X} \mathbf{1}_{(n+m) \times 1}
\end{array}\right]
$$

where $\mathbf{1}_{p \times 1}$ denotes a $p \times 1$ vector of ones. The covariance matrix of [ $\left.\mathbf{Y} \mathbf{X} \mathbf{W}\right]^{\prime}$ is

$$
\operatorname{cov}\left[\begin{array}{l}
\mathbf{Y} \\
\mathbf{X} \\
\mathbf{W}
\end{array}\right]=\left[\begin{array}{ccc}
\beta_{1}^{2} \boldsymbol{\Sigma}_{X}+\boldsymbol{\Sigma}_{\epsilon} & \beta_{1} \boldsymbol{\Sigma}_{X} & \beta_{1} \boldsymbol{\Sigma}_{X W} \\
\beta_{1} \boldsymbol{\Sigma}_{X} & \boldsymbol{\Sigma}_{X} & \boldsymbol{\Sigma}_{X W} \\
\beta_{1} \boldsymbol{\Sigma}_{\mathbf{X W}}^{\prime} & \boldsymbol{\Sigma}_{\mathbf{X W}}^{\prime} & \boldsymbol{\Sigma}_{W}
\end{array}\right]
$$

where $\boldsymbol{\Sigma}_{\mathbf{X}}$ and $\boldsymbol{\Sigma}_{\mathbf{W}}$ are the covariance matrices of $\mathbf{X}$ and $\mathbf{W}$ respectively, and $\boldsymbol{\Sigma}_{\mathbf{X W}}$ is the $n \times m$ crosscovariance matrix $E\left[\left(\mathbf{X}-\mu_{X} \mathbf{1}_{n \times 1}\right)\left(\mathbf{W}-\mu_{X} \mathbf{1}_{m \times 1}\right)^{\prime}\right]$.

If $\mathbf{X}$ had been observed, the parameter vector $\boldsymbol{\beta}=\left[\beta_{0} \beta_{1}\right]^{\prime}$ could be estimated by weighted least squares (WLS) as

$$
\hat{\boldsymbol{\beta}}_{\mathrm{WLS}}=\left(\mathbb{X}^{\prime} \boldsymbol{\Sigma}_{\boldsymbol{\epsilon}}^{-1} \mathbb{X}\right)^{-1} \mathbb{X}^{\prime} \boldsymbol{\Sigma}_{\boldsymbol{\epsilon}}^{-1} \mathbf{Y}
$$

where $\mathbb{X}=\left[\mathbf{1}_{n \times 1} \mathbf{X}\right]$ and $\boldsymbol{\Sigma}_{\boldsymbol{\epsilon}}=\operatorname{cov}(\boldsymbol{\epsilon})$. In practice, $\boldsymbol{\Sigma}_{\boldsymbol{\epsilon}}$ must be estimated.

With $\mathbf{X}$ unobserved, a natural approach is to use observations $\mathbf{W}$ to predict $\mathbf{X}$ by kriging, then use predictions $\hat{\mathbf{X}}$ in place of $\mathbf{X}$ in Equation (2). This krige-and-regress (KR) approach is developed in Section 2.

Joint normality of $[\mathbf{Y ~ X ~ W}]^{\prime}$ implies that $\mathbf{Y}$ and $\mathbf{W}$ are jointly normal and that $\mathbf{X}$ and $\mathbf{W}$ are jointly normal. Joint normality of $\mathbf{X}$ and $\mathbf{W}$ is exploited in Section 2 to obtain predictions $\hat{\mathbf{X}}$. Joint normality of $\mathbf{Y}$ and $\mathbf{W}$ can be exploited to obtain maximum likelihood (ML) estimates of the parameters. Section 3 gives details about the ML estimator.

Section 4 illustrates both KR and ML estimators using a data set obtained from the Environmental Protection Agency's (EPA) Environmental Monitoring and Assessment Program (EMAP) program. This data set is complete, i.e. both predictor and response are observed at the same locations, which allows comparison of the estimators to the WLS estimator. Section 5 describes simulation results comparing KR and ML estimators. 


\section{KRIGE AND REGRESS}

Since $\mathbf{X}$ and $\mathbf{W}$ are generated by the same spatial process, $\mathbf{X}$ can be predicted from $\mathbf{W}$ by kriging (Cressie, 1993). If the covariance matrices $\boldsymbol{\Sigma}_{\mathbf{X W}}$ and $\boldsymbol{\Sigma}_{\mathbf{W}}$ were known, this prediction would be the best linear unbiased prediction (BLUP) of $\mathbf{X}$ given $\mathbf{W}$ and would be computed as

$$
\begin{aligned}
\operatorname{BLUP}(\mathbf{X} \mid \mathbf{W}) & =\operatorname{BLUE}\left(\mu_{X}\right) \mathbf{1}_{n \times 1}+\boldsymbol{\Sigma}_{\mathbf{X W}} \boldsymbol{\Sigma}_{\mathbf{W}}^{-1}\left[\mathbf{W}-\operatorname{BLUE}\left(\mu_{X}\right) \mathbf{1}_{m \times 1}\right] \\
& =\Lambda \mathbf{W}
\end{aligned}
$$

where $\operatorname{BLUE}\left(\mu_{X}\right)$ is the best linear unbiased estimator of $\mu_{X}$ from $\mathbf{W}$,

$$
\operatorname{BLUE}\left(\mu_{X}\right)=\frac{\mathbf{1}_{1 \times m} \boldsymbol{\Sigma}_{\mathbf{W}}^{-1}}{\mathbf{1}_{1 \times m} \boldsymbol{\Sigma}_{\mathbf{W}}^{-1} \mathbf{1}_{m \times 1}} \mathbf{W}
$$

and

$$
\boldsymbol{\Lambda}=\left[\frac{\mathbf{1}_{n \times m}-\boldsymbol{\Sigma}_{\mathbf{X W}} \boldsymbol{\Sigma}_{\mathbf{W}}^{-1} \mathbf{1}_{m \times m}}{\mathbf{1}_{1 \times m} \boldsymbol{\Sigma}_{\mathbf{W}}^{-1} \mathbf{1}_{m \times 1}}+\boldsymbol{\Sigma}_{\mathbf{X W}}\right] \boldsymbol{\Sigma}_{\mathbf{W}}^{-1}
$$

In practice, $\boldsymbol{\Sigma}_{\mathbf{X W}}$ and $\boldsymbol{\Sigma}_{\mathbf{W}}$ must be estimated, usually by fitting a parametric model to the covariance exhibited by $\mathbf{W}$, the observations from the predictor process. Cressie (1993) lists some common parametric semivariogram models and describes a number of estimation methods, including method-ofmoments (MOM), weighted least squares, and restricted maximum likelihood (REML). The Gaussian assumption makes REML a natural choice, and this is what is used in Sections 4 and 5. Section 4 introduces the three-parameter exponential semivariogram model, which is the model used in Sections 4 and 5.

REML estimates can be found numerically using S-Plus, MATLAB, or a number of other software packages. The numerical procedures used require initial values for $\boldsymbol{\theta}_{X}$. A natural method for finding reasonable initial values is to plot the MoM semivariogram estimate (this can be done using S-Plus's variogram function in the spatial module), then fit a parametric function by eye by choosing a variogram function and parameters suggested by the plot, then overlaying the MoM plot with a plot of the parametric semivariogram and adjusting either the parameters or the function as necessary. This is the procedure used in Section 4.

The parametric semivariogram model can be used to model $\operatorname{cov}\left(W_{i}, W_{j}\right)$ as a function of the semivariogram parameters and the distance between the locations where $W_{i}$ and $W_{j}$ were observed. Distances between all observations are known, so once parameter estimates are found, estimates $\hat{\mathbf{\Sigma}}_{\mathbf{X W}}$ and $\hat{\mathbf{\Sigma}}_{\mathbf{W}}$ can be calculated.

With these estimates $\boldsymbol{\Lambda}$ from Equation (4), $\hat{\Lambda}$ is estimated as

$$
\hat{\boldsymbol{\Lambda}}=\left[\frac{\mathbf{1}_{n \times m}-\hat{\boldsymbol{\Sigma}}_{\mathbf{X W}} \hat{\boldsymbol{\Sigma}}_{\mathbf{W}}^{-1} \mathbf{1}_{m \times m}}{\mathbf{1}_{1 \times m} \hat{\boldsymbol{\Sigma}}_{\mathbf{W}}^{-1} \mathbf{1}_{m \times 1}}+\hat{\boldsymbol{\Sigma}}_{\mathbf{X W}}\right] \hat{\boldsymbol{\Sigma}}_{\mathbf{W}}^{-1}
$$


and predictions of $\hat{\mathbf{X}}$ are calculated as

$$
\hat{\mathbf{X}}=\hat{\Lambda} \mathbf{W}
$$

If $\boldsymbol{\Sigma}_{\boldsymbol{\epsilon}}$ were known, the KR estimator could be calculated by substituting $\hat{\mathbf{X}}$ for $\mathbf{X}$ in Equation (2). In practice, $\boldsymbol{\Sigma}_{\boldsymbol{\epsilon}}$ must be estimated, and this is not as straightforward as estimating $\boldsymbol{\Sigma}_{\mathbf{X W}}$ and $\boldsymbol{\Sigma}_{\mathbf{W}}$.

Since the $X$ vector is not observed, the REML estimate of $\boldsymbol{\Sigma}_{\boldsymbol{\epsilon}}$ is not available. Instead, estimates of quasi-residuals $\mathbf{R}=\mathbf{Y}-E(\mathbf{Y} \mid \mathbf{W})$ are obtained. Since the covariance matrix of $\mathbf{R}$ is

$$
\boldsymbol{\Sigma}_{\mathbf{R}}=\beta_{1}^{2}\left(\boldsymbol{\Sigma}_{\mathbf{X}}-\boldsymbol{\Sigma}_{\mathbf{X W}} \boldsymbol{\Sigma}_{\mathbf{W}}^{-1} \Sigma_{\mathbf{X W}}^{\prime}\right)+\boldsymbol{\Sigma}_{\boldsymbol{\epsilon}}
$$

$\boldsymbol{\Sigma}_{\boldsymbol{\epsilon}}$ can be estimated by subtracting from $\hat{\boldsymbol{\Sigma}}_{\mathbf{R}}$ an estimate of $\beta_{1}^{2}\left(\boldsymbol{\Sigma}_{\mathbf{X}}-\boldsymbol{\Sigma}_{\mathbf{X W}} \Sigma_{\mathbf{W}}^{-1} \Sigma_{\mathbf{X W}}^{\prime}\right)$.

Since $E(\mathbf{Y} \mid \mathbf{W})=\beta_{0} \mathbf{1}_{n \times 1}+\beta_{1} E(\mathbf{X} \mid \mathbf{W})$, and $E(\mathbf{X} \mid \mathbf{W})=\hat{\mathbf{X}}, \mathbf{R}$ can be estimated as $\mathbf{Y}-\hat{\mathbb{X}} \hat{\boldsymbol{\beta}}$ where $\hat{\boldsymbol{\beta}}$ is the unweighted KR estimate of $\boldsymbol{\beta}$,

$$
\hat{\boldsymbol{\beta}}=\left(\hat{\mathbb{X}}^{\prime} \hat{\mathbb{X}}\right)^{-1} \hat{\mathbb{X}}^{\prime} \mathbf{Y}
$$

and $\hat{\mathbb{X}}=\left[\mathbf{1}_{n \times 1} \hat{\mathbf{X}}\right]$.

$\boldsymbol{\Sigma}_{\mathbf{R}}$ can then be estimated by finding the REML estimates of the covariance parameters of $\hat{\mathbf{R}}$. The natural estimate of $\boldsymbol{\Sigma}_{\mathbf{X}}-\Sigma_{\mathbf{X W}} \Sigma_{\mathbf{W}}^{-1} \Sigma_{\mathbf{X W}}^{\prime}$ is calculated by substituting estimates of $\boldsymbol{\Sigma}_{\mathbf{X}}, \boldsymbol{\Sigma}_{\mathbf{X W}}$ and $\boldsymbol{\Sigma}_{\mathbf{W}}$. (Note that $\hat{\boldsymbol{\Sigma}}_{\mathbf{X}}$ is obtained from the same parameters and parametric function as $\hat{\boldsymbol{\Sigma}}_{\mathbf{X W}}$ and $\hat{\boldsymbol{\Sigma}}_{\mathbf{W}}$.) Then estimate $\boldsymbol{\Sigma}_{\boldsymbol{\epsilon}}$ by

$$
\hat{\boldsymbol{\Sigma}}_{\boldsymbol{\epsilon}}=\hat{\mathbf{\Sigma}}_{\mathbf{R}}-\hat{\beta}_{1}^{2}\left(\hat{\mathbf{\Sigma}}_{\mathbf{X}}-\hat{\mathbf{\Sigma}}_{\mathbf{X W}} \hat{\mathbf{\Sigma}}_{\mathbf{W}}^{-1} \hat{\mathbf{\Sigma}}_{\mathbf{X W}}^{\prime}\right)
$$

There is no guarantee that $\hat{\boldsymbol{\Sigma}}_{\boldsymbol{\epsilon}}$ is positive definite, or even non-negative definite. When it is not non-negative definite, the unweighted KR estimate (5) can be used. In the simulation study reported in Section 5, $\hat{\Sigma}_{\boldsymbol{\epsilon}}$ was always positive definite.

With $\hat{\boldsymbol{\Sigma}}_{\boldsymbol{\epsilon}}$ and $\hat{\mathbb{X}}$ as above, the KR estimate of $\boldsymbol{\beta}$ is

$$
\hat{\boldsymbol{\beta}}_{\mathrm{KR}}=\left(\hat{\mathbb{X}}^{\prime} \hat{\boldsymbol{\Sigma}}_{\boldsymbol{\epsilon}}^{-1} \hat{\mathbb{X}}\right)^{-1} \hat{\mathbb{X}}^{\prime} \hat{\boldsymbol{\Sigma}}_{\boldsymbol{\epsilon}}^{-1} \mathbf{Y}
$$
$\hat{\beta}_{1, \mathrm{KR}}$.

The parameter of interest is the slope parameter $\beta_{1}$. Denote the KR estimate of this parameter by It is important to note that if no spatial covariance in the predictor process is detected, i.e. $\hat{\mathbf{\Sigma}}_{\mathbf{X W}}$ is zero, then $\hat{\mathbf{X}}=\overline{\mathbf{W}} \mathbf{1}_{m \times 1}$, so $\hat{\mathbb{X}}$ is not full rank and $\hat{\mathbb{X}}^{\prime} \hat{\boldsymbol{\Sigma}}_{\boldsymbol{\epsilon}}^{-1} \hat{\mathbb{X}}$ is not invertible. This is sensible, since if $\hat{\boldsymbol{\Sigma}}_{\mathbf{X W}}$ is zero, then $\mathbf{W}$ gives no information about $\mathbf{X}$ other than its mean and cannot be used to estimate $\boldsymbol{\beta}$. 


\subsection{Estimating the variance of $\hat{\beta}_{1, \mathrm{KR}}$}

In keeping with the KR paradigm of substituting $\hat{\mathbf{X}}$ for $\mathbf{X}$ in the WLS formula (2), a natural variance estimator for $\hat{\boldsymbol{\beta}}_{\mathrm{KR}}$ is

$$
\widehat{\operatorname{var}}_{\text {naïve }}=\left(\hat{\mathbb{X}}^{\prime} \hat{\boldsymbol{\Sigma}}_{\epsilon}^{-1} \hat{\mathbb{X}}\right)^{-1}
$$

In the simulation study described in Equation (5) this naïve estimator performs well. However, it ignores the error in $\hat{\mathbf{X}}$ and may not be reliable in general.

A more accurate variance estimator can be developed as follows. Suppose the covariance matrices $\boldsymbol{\Sigma}_{\mathbf{W}}, \boldsymbol{\Sigma}_{\mathbf{X W}}$ and $\boldsymbol{\Sigma}_{\boldsymbol{\epsilon}}$ are known. Let

$$
\tilde{\boldsymbol{\beta}}_{\mathrm{KR}}=\left(\mathbf{W}^{\prime} \boldsymbol{\Lambda}^{\prime} \boldsymbol{\Sigma}_{\boldsymbol{\epsilon}} \boldsymbol{\Lambda} \mathbf{W}\right)^{-1} \boldsymbol{\Lambda} \mathbf{W}^{\prime} \boldsymbol{\Sigma}_{\boldsymbol{\epsilon}} \mathbf{Y}
$$

the KR estimator with known covariances. Then $\tilde{\beta}_{1, \mathrm{KR}}$ can be written as

$$
\tilde{\beta}_{1, \mathrm{KR}}=\frac{\mathbf{W}^{\prime} \mathbf{\Lambda}^{\prime} \mathbf{M Y}}{\mathbf{W}^{\prime} \mathbf{\Lambda}^{\prime} \mathbf{M} \Lambda \mathbf{W}}
$$

where $\mathbf{M}=\mathbf{1}_{1 \times \mathbf{n}} \boldsymbol{\Sigma}_{\boldsymbol{\epsilon}}{ }^{-1} \mathbf{1}_{\mathrm{n} \times \mathbf{1}} \boldsymbol{\Sigma}_{\boldsymbol{\epsilon}}{ }^{-1}-\boldsymbol{\Sigma}_{\boldsymbol{\epsilon}}{ }^{-1} \mathbf{1}_{\mathrm{n} \times \mathbf{n}} \boldsymbol{\Sigma}_{\boldsymbol{\epsilon}}{ }^{-1}$.

The variance of the expression in Equation (8) can be calculated by first conditioning on $\mathbf{W}$ :

$$
\begin{aligned}
\operatorname{var}\left(\tilde{\beta}_{1, \mathrm{KR}}\right) & =E\left\{\operatorname{var}\left(\tilde{\beta}_{1, \mathrm{KR}} \mid \mathbf{W}\right)\right\}+\operatorname{var}\left\{E\left(\tilde{\beta}_{1, \mathrm{KR}} \mid \mathbf{W}\right)\right\} \\
& =E\left(Q_{1} Q_{2}^{-2}\right)+E\left(Q_{3} Q_{2}^{-2}\right)-\left[E\left(Q_{3} Q_{2}^{-1}\right)\right]^{2}
\end{aligned}
$$

where

$$
\begin{aligned}
& Q_{1}=\mathbf{W}^{\prime} \boldsymbol{\Lambda}^{\prime} \mathbf{M}\left[\boldsymbol{\Sigma}_{\boldsymbol{\epsilon}}+\beta_{1}^{2}\left(\boldsymbol{\Sigma}_{\mathbf{X}}-\Sigma_{\mathbf{X W}} \boldsymbol{\Sigma}_{\mathbf{W}}^{-1} \boldsymbol{\Sigma}_{\mathbf{X W}}^{\prime}\right)\right] \mathbf{M}^{\prime} \boldsymbol{\Lambda} \mathbf{W} \\
& Q_{2}=\mathbf{W}^{\prime} \boldsymbol{\Lambda}^{\prime} \mathbf{M} \mathbf{\Lambda} \mathbf{W} \\
& Q_{3}=\mathbf{W}^{\prime} \boldsymbol{\Lambda}^{\prime} \mathbf{M} \boldsymbol{\Sigma}_{\mathbf{X W}} \boldsymbol{\Sigma}_{\mathbf{W}}^{-1} \mathbf{W}-\mu_{X} \mathbf{W}^{\prime} \boldsymbol{\Lambda}^{\prime} \mathbf{M} \boldsymbol{\Sigma}_{\mathbf{X W}} \boldsymbol{\Sigma}_{\mathbf{W}}^{-1} \mathbf{1}_{m \times 1}
\end{aligned}
$$

These calculations follow from

$$
\begin{aligned}
E(\mathbf{Y} \mid \mathbf{W}) & =\beta_{0} \mathbf{1}_{n \times 1}+\beta_{1}\left\{\mu_{X} \mathbf{1}_{n \times 1}+\boldsymbol{\Sigma}_{\mathbf{X W}} \boldsymbol{\Sigma}_{\mathbf{W}}^{-1}\left(\mu_{X} \mathbf{1}_{m \times 1}-\mathbf{W}\right)\right\} \\
\operatorname{var}(\mathbf{Y} \mid \mathbf{W}) & =\boldsymbol{\Sigma}_{\boldsymbol{\epsilon}}+\beta_{1}^{2}\left(\boldsymbol{\Sigma}_{\mathbf{X}}-\boldsymbol{\Sigma}_{\mathbf{X W}} \boldsymbol{\Sigma}_{\mathbf{W}}^{-1} \boldsymbol{\Sigma}_{\mathbf{X W}}^{\prime}\right)
\end{aligned}
$$

and the fact that $\mathbf{M} \mathbf{1}_{n \times 1}=\mathbf{0}$.

$Q_{1}, Q_{2}$ and $Q_{3}$ can be estimated by substituting the above-described estimates of $\boldsymbol{\Sigma}_{\mathbf{W}}, \boldsymbol{\Sigma}_{\mathbf{X W}}$ and $\Sigma_{\boldsymbol{\epsilon}}$. The natural estimate of $\mu_{X}$ is

$$
\hat{\mu}_{X}=\frac{\mathbf{1}_{1 \times m} \hat{\boldsymbol{\Sigma}}_{\mathbf{W}}^{-1}}{\mathbf{1}_{1 \times m} \hat{\boldsymbol{\Sigma}}_{\mathbf{W}}^{-1} \mathbf{1}_{m \times 1}} \mathbf{W}
$$

which is Equation (3) with $\boldsymbol{\Sigma}_{\mathbf{W}}$ estimated. $\hat{\boldsymbol{\beta}}_{1, \mathrm{KR}}$ estimates $\boldsymbol{\beta}_{1}$ in estimating $Q_{1}$. 
The expectations in Equation (9) can be estimated by Monte Carlo integration as follows:

1. Repeatedly generate random vectors $\hat{\mathbf{W}}$ from a normal $\left(\hat{\mu}_{X} \mathbf{1}_{m \times 1}, \hat{\boldsymbol{\Sigma}}_{\mathbf{W}}\right)$ distribution, where $\hat{\mu}_{X}$ is from Equation (13) and $\boldsymbol{\Sigma}_{\mathbf{W}}$ has been estimated from the observed $\mathbf{X}$ vector (e.g. via REML, as described above).

2. For each $\hat{\mathbf{W}}$ generated, find $\hat{Q}_{1}, \hat{Q}_{2}$ and $\hat{Q}_{3}$, estimates of $Q_{1}, Q_{2}$ and $Q_{3}$, by substituting estimates for the parameters in Equation (10), (11) and (12), as described above.

3. Calculate the averages of $\hat{Q}_{1} \hat{Q}_{2}^{-2}, \hat{Q}_{3} \hat{Q}_{2}^{-2}$ and $\hat{Q}_{3} \hat{Q}_{2}^{-1}$ over all Wis generated.

Thus an alternative variance estimator to the naïve estimator (7) is

$$
\widehat{\operatorname{var}}\left(\hat{\beta}_{1, \mathrm{KR}}\right)=\hat{E}\left(Q_{1} Q_{2}^{-2}\right)+\hat{E}\left(Q_{3} Q_{2}^{-2}\right)-\left[\hat{E}\left(Q_{3} Q_{2}^{-1}\right)\right]^{2}
$$

The performance of this variance estimator is illustrated in Sections 4 and 5 .

\section{MAXIMUM LIKELIHOOD}

The assumptions described in Section 1 imply

$$
\left[\begin{array}{l}
\mathbf{Y} \\
\mathbf{W}
\end{array}\right] \sim N\left(\left[\begin{array}{c}
\left(\beta_{0}+\beta_{1} \mu_{X}\right) \mathbf{1}_{n \times 1} \\
\mu_{X} \mathbf{1}_{m \times 1}
\end{array}\right],\left[\begin{array}{cc}
\beta_{1}^{2} \boldsymbol{\Sigma}_{\mathbf{X}}+\boldsymbol{\Sigma}_{\boldsymbol{\epsilon}} & \beta_{1} \boldsymbol{\Sigma}_{\mathbf{X W}} \\
\beta_{1} \boldsymbol{\Sigma}_{\mathbf{X W}}^{\prime} & \boldsymbol{\Sigma}_{\mathbf{W}}
\end{array}\right]\right)
$$

The joint density of $Y$ and $W$ is therefore

$$
f_{Y W}(y, w)=\frac{1}{\sqrt{2 \pi|\mathbf{\Sigma}|}} \exp \left(-\frac{1}{2} \mathbf{V}^{\prime} \boldsymbol{\Sigma}^{-1} \mathbf{V}\right)
$$

where

$$
\boldsymbol{\Sigma}=\left[\begin{array}{ll}
\beta_{1}^{2} \boldsymbol{\Sigma}_{\mathbf{X}}+\boldsymbol{\Sigma}_{\boldsymbol{\epsilon}} & \beta_{1} \boldsymbol{\Sigma}_{\mathbf{X W}} \\
\beta_{1} \boldsymbol{\Sigma}_{\mathbf{X W}}^{\prime} & \boldsymbol{\Sigma}_{\mathbf{W}}
\end{array}\right]
$$

and

$$
\begin{aligned}
\mathbf{V} & =\left[\begin{array}{l}
\mathbf{V}_{\mathbf{Y}} \\
\mathbf{V}_{\mathbf{X}}
\end{array}\right] \\
& =\left[\begin{array}{c}
\mathbf{Y}-\left(\beta_{0}+\beta_{1} \mu_{X}\right) \mathbf{1}_{n \times 1} \\
\mathbf{W}-\mu_{X} \mathbf{1}_{m \times 1}
\end{array}\right]
\end{aligned}
$$

In Section 2, $\boldsymbol{\Sigma}_{\mathbf{X W}}$ and $\boldsymbol{\Sigma}_{\mathbf{W}}$ were estimated by assuming a parametric semivariogram function for the predictor process, so that these unknown matrices are functions of a vector of unknown parameters. Maximum likelihood estimation requires that the unknown $\boldsymbol{\Sigma}_{\boldsymbol{\epsilon}}$ is also determined by a vector of 
parameters. Let $\boldsymbol{\theta}_{X}$ and $\boldsymbol{\theta}_{\epsilon}$ denote the vectors of semivariogram parameters of the predictor and error processes, respectively. The maximum likelihood estimate of $\boldsymbol{\beta}$ is found by minimising

$$
l(\boldsymbol{\phi})=-\log \left(f_{Y W}\right)=\frac{1}{2} \log \operatorname{det}(\boldsymbol{\Sigma})+\frac{1}{2} \mathbf{V}^{\prime} \boldsymbol{\Sigma}^{-1} \mathbf{V}
$$

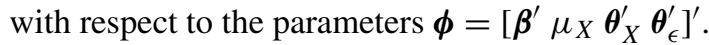

Typically, $\boldsymbol{\theta}_{X}$ and $\boldsymbol{\theta}_{\epsilon}$ are each of length 3, so minimisation of Equation (15) is a problem in nine-dimensional space. The minimisation is a straightforward constrained optimisation problem (the semivariogram parameters must be positive) that can be accomplished numerically. Estimation in Sections 4 and 5 is done using Matlab.

Numerical optimisation functions require initial values for all parameters. Obvious initial values for $\boldsymbol{\beta}$ and $\mu_{X}$ are $\boldsymbol{\beta}_{\mathrm{KR}}$ and $\hat{\mu}_{X}$, respectively. REML estimates of $\boldsymbol{\theta}_{X}$ obtained using $\mathbf{W}$ can be used as initial values for $\boldsymbol{\theta}_{X}$.

Obtaining reasonable initial values for $\boldsymbol{\theta}_{\epsilon}$ is more complicated. Equation (6) gives the estimate of $\boldsymbol{\Sigma}_{\boldsymbol{\epsilon}}$ used to calculate $\hat{\boldsymbol{\beta}}_{\mathrm{KR}}$. As noted in Section 2, $\hat{\boldsymbol{\Sigma}}_{\boldsymbol{\epsilon}}$ may not be positive definite. Furthermore, it is unlikely that it will resemble a matrix obtained from a parametric semivariogram function of distance. However, given $\hat{\boldsymbol{\Sigma}}_{\boldsymbol{\epsilon}}$, the distances and the parametric function, useful initial values for $\boldsymbol{\theta}_{\epsilon}$ may be obtained by solving the elements of $\hat{\boldsymbol{\Sigma}}_{\boldsymbol{\epsilon}}$ for $\boldsymbol{\theta}_{\boldsymbol{\epsilon}}$. Details for the exponential semivariogram model are given in Section 4.

The result of the numerical optimisation is a vector of ML estimates for all nine parameters:

$$
\hat{\boldsymbol{\phi}}_{\mathrm{ML}}=\left[\begin{array}{llll}
\boldsymbol{\beta}_{\mathrm{ML}}^{\prime} & \hat{\mu}_{X, \mathrm{ML}} & \hat{\boldsymbol{\theta}}_{X, \mathrm{ML}}^{\prime} & \hat{\boldsymbol{\theta}}_{\epsilon, \mathrm{ML}}^{\prime}
\end{array}\right]^{\prime}
$$

and the ML estimate of the slope parameter $\beta_{1}$ is the second element of $\hat{\boldsymbol{\phi}}$ :

$$
\hat{\beta}_{1, \mathrm{ML}}=\hat{\phi}_{2, \mathrm{ML}}
$$

As with the KR estimator, problems occur when there is a lack of spatial dependence in $\mathbf{W}$. When $\Sigma_{\mathbf{X W}} \approx \mathbf{0}$, the negative log-likelihood $l$ cannot be minimised uniquely for $\beta_{1}$.

\subsection{Estimating the variance of $\hat{\beta}_{1, \mathrm{ML}}$}

If the distribution of [Y W]' satisfies a number of regularity conditions (see, e.g. Lehmann, 1998), which in the Gaussian case essentially depend on the form of the semivariogram functions, then the ML estimator is asymptotically normal with mean equal to the true parameter vector. The asymptotic covariance matrix of $\hat{\boldsymbol{\phi}}_{\mathrm{ML}}$ is the inverse of the Fisher information matrix $\mathcal{I}(\phi)^{-1}$ where the $i j$ th element of $\mathcal{I}(\phi)$ is

$$
\mathcal{I}(\boldsymbol{\phi})_{i j}=E\left(\frac{\partial l}{\partial \phi_{i}} \frac{\partial l}{\partial \phi_{j}}\right)=-E\left(\frac{\partial l^{2}}{\partial \phi_{i} \phi_{j}}\right)
$$

evaluated at the true value of $\boldsymbol{\phi}$. The variance of $\hat{\beta}_{1, \mathrm{ML}}$ can be estimated as

$$
\widehat{\operatorname{var}}\left(\hat{\beta}_{1, \mathrm{ML}}\right)=\left.\mathcal{I}^{-1}(\boldsymbol{\phi})_{22}\right|_{\boldsymbol{\phi}=\hat{\boldsymbol{\phi}}_{\mathrm{ML}}}
$$


The asymptotic variance used here depends on an increasing number of independent identically distributed (iid) vectors [Y W $]^{\prime}$. In most applications, such as the one presented in Section 4, only one vector $[\mathbf{Y ~ W}]^{\prime}$ has been observed, so the variance estimator (17) may not perform very well. Simulations in Section 5 indicate that Equation (17) is much too small, leading to inference that is much too optimistic

\section{EXAMPLE}

The data set used in this example is a subset of data obtained from the EPA's EMAP program. Observations on rivers and streams in the Mid-Altantic region were taken, and EPA's goal was to relate stream characteristics with land use variables (Herlihy et al., 1998). The variables used below are chloride concentrations (cl) and per cent of the watershed in forest (for.nlcd). A log transformation of $\mathrm{cl}$ and a logit transformation of for.nlcd give variables that look approximately normal and approximately linearly related. Some of the sites were visited more than once, so the data set was edited to include only the initial observation per site, leaving 558 observations. These initial visits occurred over three consecutive years, so there may be temporal correlation in addition to the spatial correlation, but this aspect of the data was ignored in this example. There was no evidence of anisotropy in variogram plots of the data, so we assume that the spatial correlation is isotropic.

The assumed model is

$$
Y_{i}=\beta_{0}+\beta_{1} X_{i}+\epsilon_{i}
$$

for the $i$ th site, where $Y_{i}=\log (\mathrm{cl})_{i}$ and $X_{i}=\operatorname{logit}(\text { for.nlcd })_{i}$, and

$$
\boldsymbol{\epsilon} \sim N\left(\mathbf{0}, \boldsymbol{\Sigma}_{\boldsymbol{\epsilon}}\right)
$$

Using the complete data set, the weighted least squares estimate of $\beta_{1}$ can be obtained as in Equation (2), once an estimate of $\boldsymbol{\Sigma}_{\boldsymbol{\epsilon}}$ has been found.

To estimate $\boldsymbol{\Sigma}_{\boldsymbol{\epsilon}}$, the residuals $\boldsymbol{e}$ from an unweighted least squares regression were calculated:

$$
\boldsymbol{e}=\mathbf{Y}-\mathbb{X}\left(\mathbb{X}^{\prime} \mathbb{X}\right)^{-1} \mathbb{X}^{\prime} \mathbf{Y}
$$

where $\mathbb{X}=\left[\mathbf{1}_{558 \times 1} \mathbf{X}\right]$. The MoM variogram estimator of $\boldsymbol{e}$ was plotted in order to select a semivariogram model and starting parameter values to estimate $\boldsymbol{\Sigma}_{\boldsymbol{\epsilon}}$ by REML. An exponential model appears to fit, so the $i j$ th element of $\boldsymbol{\Sigma}_{\boldsymbol{\epsilon}}$ is

$$
\operatorname{cov}\left(\epsilon_{i}, \epsilon_{j}\right)= \begin{cases}\theta_{\epsilon, 1}+\theta_{\epsilon, 2} & h_{i j}=0 \\ \theta_{\epsilon, 2} \exp \left(-h_{i j} / \theta_{\epsilon, 3}\right) & h_{i j} \neq 0\end{cases}
$$

where $h_{i j}$ denotes the distance between the location of points $i$ and $j$. A rough fit to the MoM plot can be found using $\boldsymbol{\theta}_{\epsilon}=\left[\begin{array}{lll}0.4 & 0.4 & 2\end{array}\right]^{\prime}$. If these values are used as initial values in a REML optimisation, the result is an estimated $\boldsymbol{\theta}_{\epsilon}$ of [0.49 0.311 .63$]^{\prime}$, which then can be used to calculate $\hat{\boldsymbol{\Sigma}}_{\boldsymbol{\epsilon}}$ according to Equation (20). 
The WLS estimate of $\boldsymbol{\beta}$ is

$$
\hat{\boldsymbol{\beta}}_{\mathrm{WLS}}=\left(\mathbb{X}^{\prime} \hat{\boldsymbol{\Sigma}}_{\boldsymbol{\epsilon}}^{-1} \mathbb{X}\right)^{-1} \mathbb{X}^{\prime} \hat{\boldsymbol{\Sigma}}_{\boldsymbol{\epsilon}}^{-1} Y=\left[\begin{array}{l}
5.16 \\
-0.38
\end{array}\right]
$$

The estimated variance of $\hat{\beta}_{\mathrm{WLS}, 1}$ is the $(2,2)$ entry in $\left(\mathbb{X}^{\prime} \hat{\boldsymbol{\Sigma}}_{\boldsymbol{\epsilon}}^{-1} \mathbb{X}\right)^{-1}$, which gives a standard error of

$$
\operatorname{SE}\left(\hat{\beta}_{\mathrm{WLS}, 1}\right)=0.0116
$$

To check the performance of KR and ML on misaligned data, the 558 locations in the data set are randomly split into two equal halves. The covariate $X_{i}=\operatorname{logit}($ for.nlcd) is assumed to have been observed on one half, and the response $Y_{i}=\log (\mathrm{cl})$ is assumed to have been observed on the other. The KR and ML estimates, along with their standard errors, are calculated as described in Sections 2 and 3. The random splitting was repeated 250 times. The performance of the estimators can then be compared with each other.

The ML and KR estimators can also be compared to the WLS estimate calculated by taking the response vector from the random half of the data set where $X_{i}$ are assumed to have been observed. The weighting matrix $\hat{\boldsymbol{\Sigma}}_{\boldsymbol{\epsilon}}^{-1}$ was estimated by REML using the aligned response vector. The estimate of $\beta$ obtained by WLS, can then be compared to the KR and ML estimates obtained from the misaligned data.

An example of a random splitting of the locations is shown in Figure 1. It should be noted that some of the points which are to be kriged are outside the convex hull of the $W$ 's, which may make kriging inappropriate. In this example and in the simulation study in Section 5, we assume stationarity and joint normality between $\mathbf{W}$ and $\mathbf{X}$, regardless of the locations, so that predicting $\hat{\mathbf{X}}$ by kriging is valid.

In order to automate the estimation of the KR and ML estimators, it was necessary to fix initial values for the REML estimation of $\boldsymbol{\theta}_{\mathbf{X}}$. This initial value of [ $\left[\begin{array}{ll}2 & 2\end{array}\right]^{\prime}$ was found by plotting the MoM estimate of the variogram from the complete vector of $X$ values, and fitting an exponential variogram model by eye. Similarly, the REML estimation of $\boldsymbol{\Sigma}_{\mathbf{R}}$ was given an initial value of [0.4 0.42$]^{\prime}$, the rough fit to the MoM plot of the residuals of the WLS regression described above. A small simulation study shows that results using these initial values are nearly the same as if the initial values were determined by fitting a variogram by eye to a plot using only a random half of the data.

As suggested in Section 3, the initial value of $\boldsymbol{\theta}_{\boldsymbol{\epsilon}}$ for the ML optimisation was found by manipulating the elements of $\hat{\boldsymbol{\Sigma}}_{\boldsymbol{\epsilon}}$. For notational convenience in the following, let $\boldsymbol{\Sigma}$ denote $\boldsymbol{\Sigma}_{\boldsymbol{\epsilon}}$. Assume $\boldsymbol{\Sigma}$ is given by an exponential semivariogram model as in Equation (20). (Note that $\Sigma$ is $279 \times 279$, since the 558 locations have been split.) Suppose $\epsilon_{i_{1}}$ and $\epsilon_{j_{1}}$ are the errors at locations separated by a distance of $h_{1}$, and $\epsilon_{i_{2}}$ and $\epsilon_{j_{2}}$ are the errors at locations separated by a distance of $h_{2}$. If $h_{1} \neq h_{2}$, then

$$
\begin{aligned}
\theta_{\epsilon, 3} & =\frac{\log \left(\Sigma_{i_{1} j_{1}} / \Sigma_{i_{2} j_{2}}\right)}{h_{2}-h_{1}} \\
\theta_{\epsilon, 2} & =\frac{\Sigma_{i_{1} j_{1}}}{\exp \left(-h_{1} / \theta_{\epsilon, 3}\right)}
\end{aligned}
$$




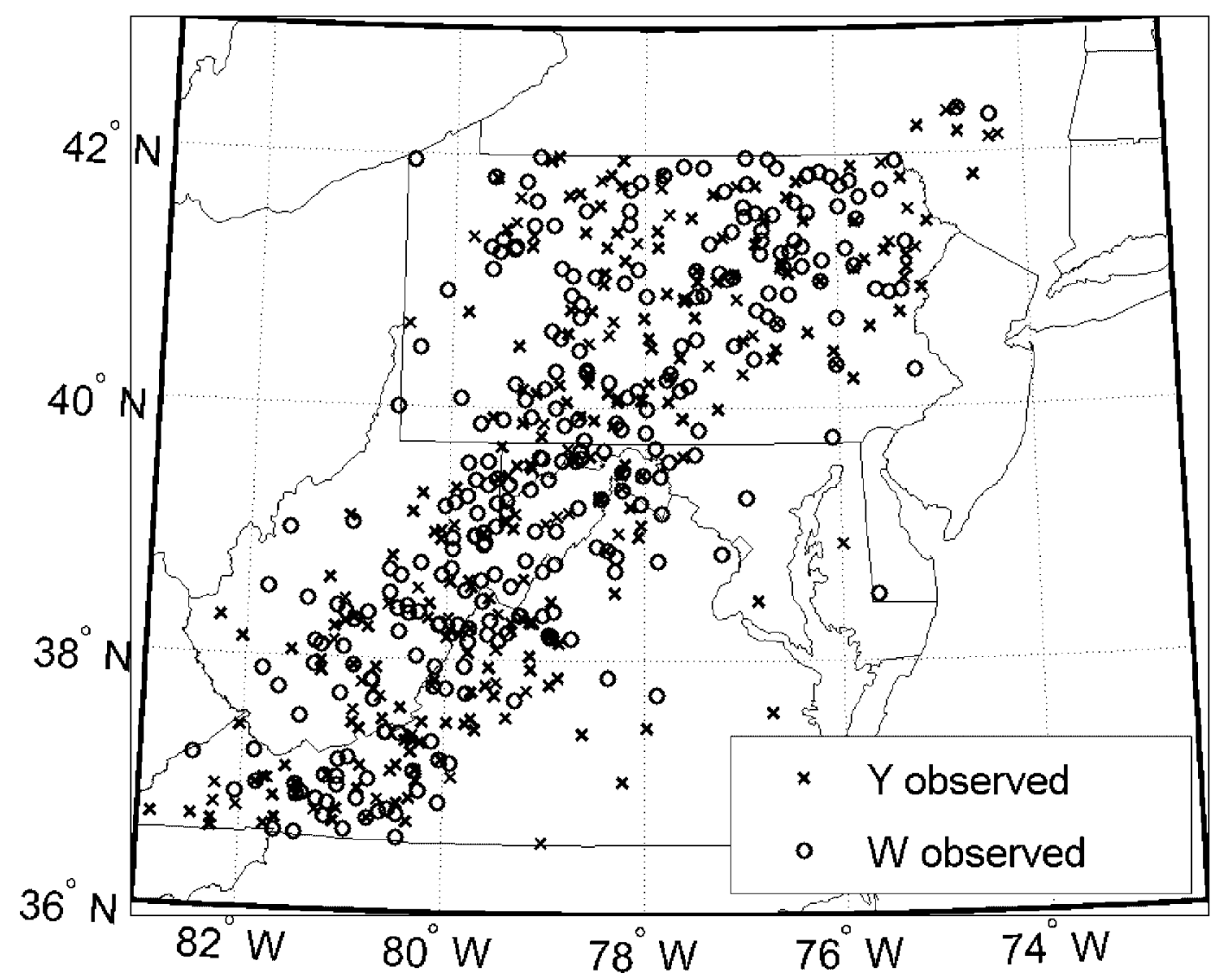

Figure 1. An illustration of randomly splitting the rivers data locations to simulate misaligned data

If $\Sigma_{i i}$ is a diagonal element, then

$$
\theta_{\epsilon, 1}=\Sigma_{i i}-\theta_{\epsilon, 2}
$$

When $\hat{\boldsymbol{\Sigma}}=\hat{\boldsymbol{\Sigma}}_{\boldsymbol{\epsilon}}$ is given as in Equation (6), different choices of $i_{1}, j_{1}, i_{2}, j_{2}$ and $i$ will give different solutions for $\boldsymbol{\theta}_{\boldsymbol{\epsilon}}$. In order to get reasonable values, solutions are found for several choices of $i$ and $j$, and the solutions are averaged. To avoid numerical problems, elements of $\Sigma$ are chosen to meet the following four criteria: (1) distances $h_{i}$ and $h_{j}$ are between 1 and 5; (2) the $\Sigma_{i j}$ are positive; (3) $\log \left(\Sigma_{i_{1} j_{1}} / \Sigma_{i_{2} j_{2}}\right) /\left(h_{2}-\right.$ $\left.h_{1}\right)>1.5 \times 10^{-8}$ and (4) $\exp \left(h_{i} \theta_{\epsilon, 3}\right)>1.5 \times 10^{-8}$. (The value $1.5 \times 10^{-8}$ is the square root of the smallest floating point number representable on the computer used for the simulations.) For this example, approximately $15000 h_{i}, h_{j}$ pairs were used to find $\theta_{\epsilon, 2}$ and approximately 7000 elements were used to find $\theta_{\epsilon, 3}$. All 279 diagonal elements of $\hat{\boldsymbol{\Sigma}}$ were used to find $\theta_{\epsilon, 1}$.

To verify that these starting values for $\boldsymbol{\theta}_{\epsilon}$ give reasonable results, a small simulation study was done to compare ML estimates when starting values for $\boldsymbol{\theta}_{\epsilon}$ were found as described and when starting values were set equal to the true parameters. ML estimates of $\beta_{1}$ were nearly identical for both sets of initial values, unless the ML estimates of $\boldsymbol{\theta}_{X}$ or $\boldsymbol{\theta}_{\epsilon}$ were very large (e.g. > 100) or very small (e.g. 


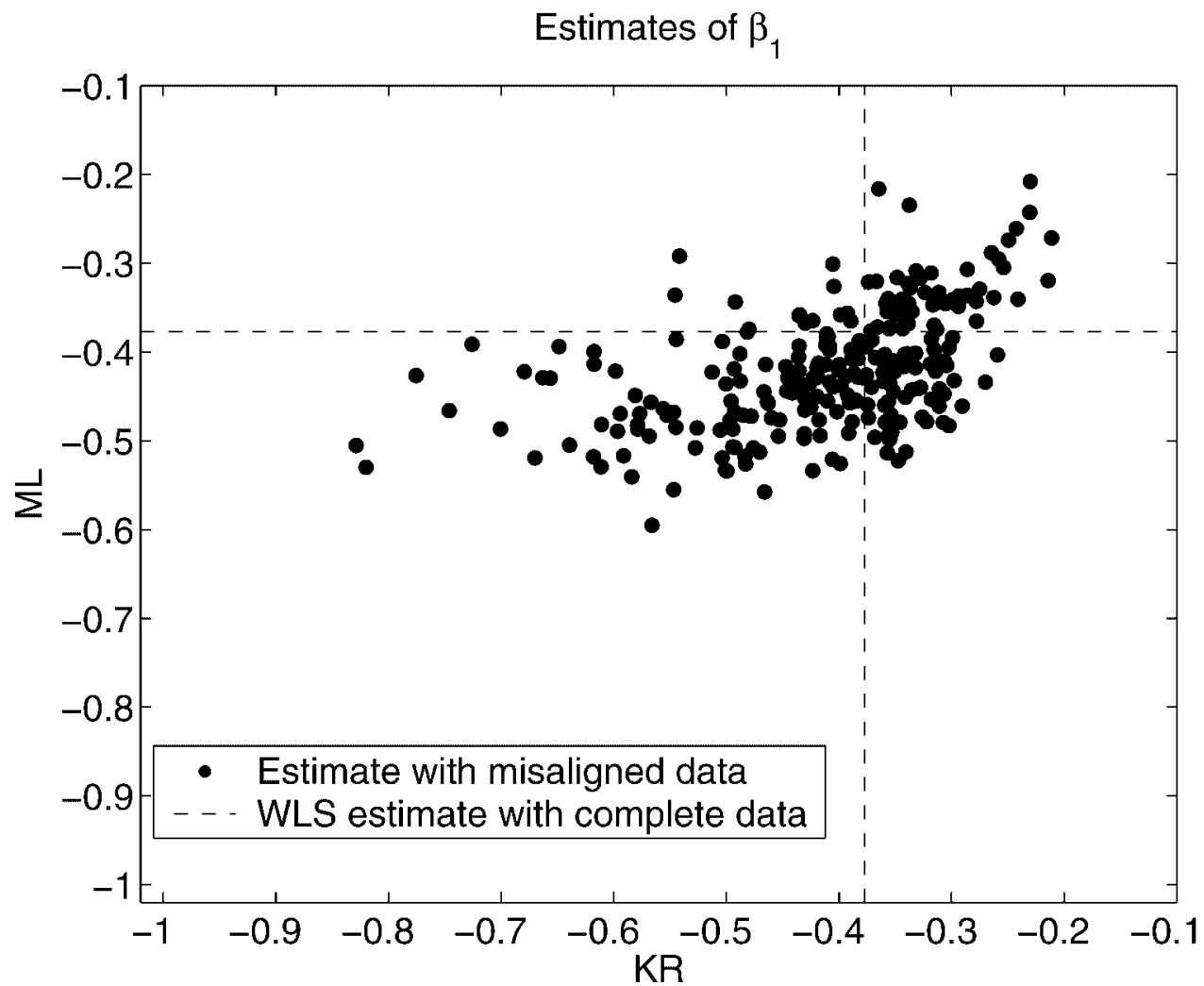

Figure 2. Scatterplot of $250 \mathrm{ML}$ vs. KR estimates of $\beta_{1}$ calculated on the randomly split rivers data. The ML estimates have a mean of -0.42 and a variance of 0.0046 . The KR estimates have a mean of -0.414 and a variance of 0.0124 . The WLS estimate is shown as dotted vertical and horizontal lines

$<0.00001)$. The very small values are close to the positivity constraint on the covariance parameters, and occasionally the optimisation function will 'get stuck' near the boundary, particularly if the initial values were close to the boundary. In practice, the covariance parameter estimates should be checked, and if they seem suspiciously large or small, several different initial values should be tried to ensure that the true maximum of the observed likelihood function is being found.

Figure 2 shows a plot of ML estimates of $\beta_{1}$ vs. KR estimates. The KR estimator is more variable; the variance of the $250 \mathrm{KR}$ estimates is 0.0124 whereas the variance of the ML estimates is only 0.0046 . Both estimators have similar means. The mean of the KR estimator is -0.414 and the mean of the ML estimator is -0.42 .

Table 1 compares ML and KR nominal 95\% confidence intervals of $\beta_{1}$

$$
\begin{aligned}
& \hat{\beta}_{1, \mathrm{ML}} \pm 1.96 \sqrt{\widehat{\operatorname{var}}\left(\hat{\beta}_{1, \mathrm{ML}}\right)} \\
& \hat{\beta}_{1, \mathrm{KR}} \pm 1.96 \sqrt{\widehat{\operatorname{var}}\left(\hat{\beta}_{1, \mathrm{KR}}\right)}
\end{aligned}
$$


Table 1. Average lengths and number of KR and ML nominal 95\% confidence intervals (out of 250) that do not overlap with the WLS intervals for the example of Section 4. The length of the WLS interval (calculated from the full data set) is 0.0455

\begin{tabular}{lcc}
\hline Method & Average length & Number of non-overlaps \\
\hline Maximum likelihood & 0.126 & 85 \\
Krige and regress & 0.509 & 0 \\
\hline
\end{tabular}

with the $95 \%$ WLS confidence interval calculated from the aligned data at all locations where $\mathbf{X}$ is assumed to have been observed for the KR and ML estimation. All of the $250 \mathrm{KR}$ intervals overlap with the WLS intervals. Only 133 out of 250 ML intervals overlap with the WLS intervals, suggesting that the ML intervals are likely too narrow. This is confirmed by noting that the average estimated variance for the ML estimator is 0.001 , much smaller than the observed variance of 0.0046 . The average estimated KR variance is 0.0186 , close to the observed variance of 0.0124 .

These results suggest that, while the ML point estimator is preferable to the KR point estimator, KR has a reasonable variance estimator whereas the ML variance estimator is too small. This hypothesis is explored in Section 5.

\section{SIMULATIONS}

This section gives results of a simulation study where data similar to the rivers data were simulated, and KR and ML estimates of $\beta_{1}$ were found. The following simulation was iterated 350 times.

Using the 558 locations from the rivers data of Section 4, a vector $\mathbf{X}_{f}$ (the subscript denotes the full set of 558 locations) was simulated with

$$
\mathbf{X} \sim N\left(\mu_{X} \mathbf{1}_{558 \times 1}, \mathbf{\Sigma}_{\mathbf{X}}\right)
$$

where $\boldsymbol{\Sigma}_{\mathbf{X}}$ is determined by the exponential semivariogram model (20) depending on the parameters $\boldsymbol{\theta}_{X}$. Table 2 lists the values of the parameters.

As described in Section 4, the 558 locations were randomly split into two halves. Accordingly, $\mathbf{X}_{f}$ was split into $\mathbf{X}$ and $\mathbf{W}$, each of length 279 .

An error vector $\boldsymbol{\epsilon}$ was generated with

$$
\boldsymbol{\epsilon} \sim N\left(\mathbf{0}_{279 \times 1}, \boldsymbol{\Sigma}_{\boldsymbol{\epsilon}}\right)
$$

Table 2. Values of the parameters used in the simulation study of Section 5. These values are obtained by estimating

\begin{tabular}{|c|c|}
\hline Parameter & Value \\
\hline$\beta$ & {$[5.08-0.3737]^{\prime}$} \\
\hline$\mu_{X}$ & 1.9 \\
\hline$\theta_{X}$ & {$\left[\begin{array}{lll}2 & 2 & 5\end{array}\right]^{\prime}$} \\
\hline$\theta_{\epsilon}$ & {$\left[\begin{array}{lll}0.5 & 0.3 & 3.33\end{array}\right]$} \\
\hline
\end{tabular}
the parameters from the data used in the example of Section 4 


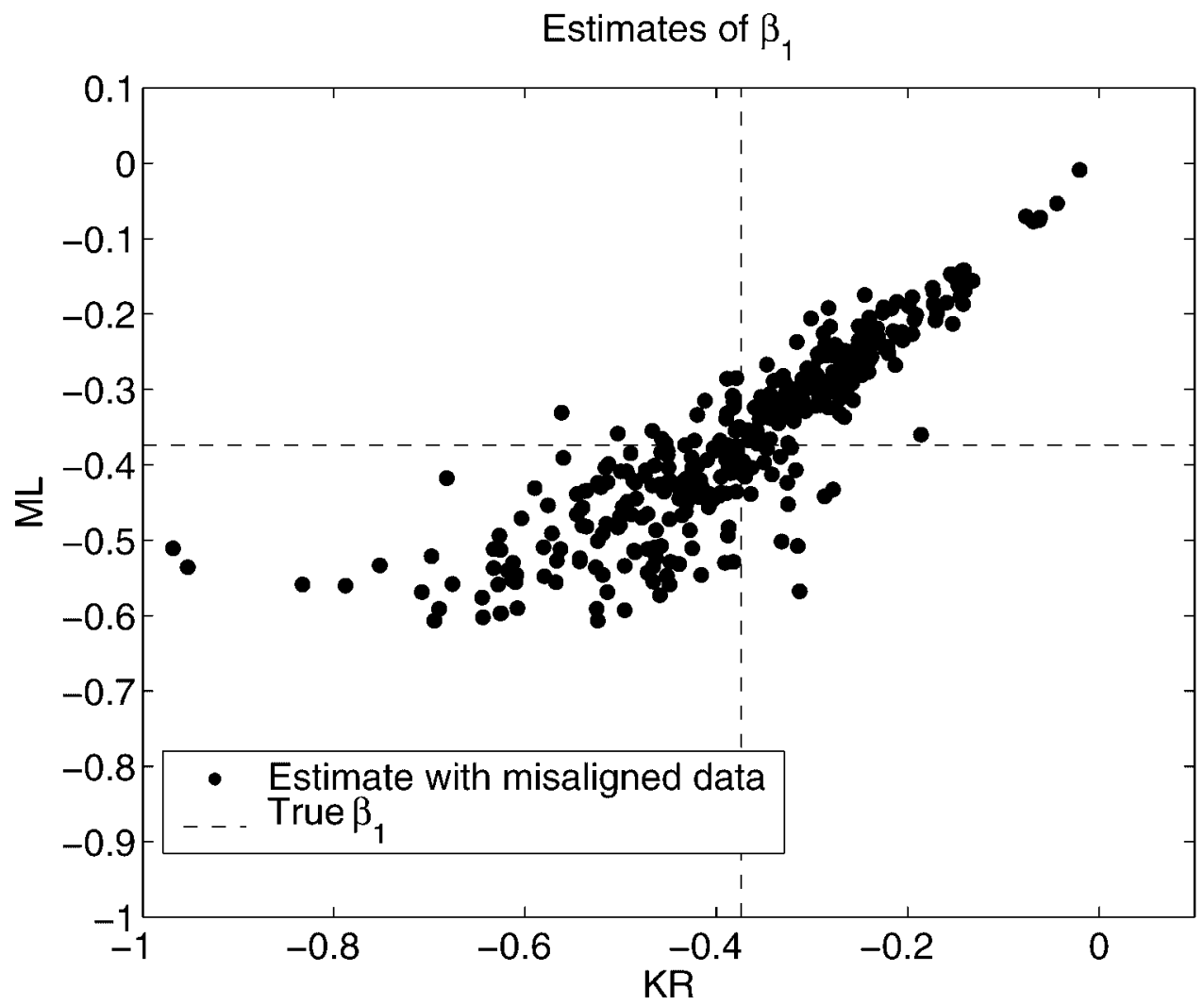

Figure 3. Scatterplot of ML vs. KR estimates of $\beta_{1}=-0.3737$ from simulated data. KR estimates are more variable than the ML estimates. The MSE of the KR estimates is approximately 0.021 whereas the MSE of the ML estimates is only about 0.015 . Both estimators are approximately unbiased. The mean of the ML estimates is -0.3661 , and the mean of the KR estimates is $-0.3782$

where $\boldsymbol{\Sigma}_{\boldsymbol{\epsilon}}$ is determined by the exponential semivariogram model (20) depending on the parameters $\boldsymbol{\theta}_{\epsilon}$, which are given in Table 2. Finally, $\mathbf{Y}$ was calculated according to model (1) where $\boldsymbol{\beta}$ is also given in Table 2.

From the simulated data $[\mathbf{Y ~ W}]^{\prime}, \mathrm{KR}$ and ML estimates of $\beta_{1}$ were calculated as described in Section 4. The ML variance estimate (17) and the KR variance estimates (7) and (14) were also calculated.

Figure 3 shows a plot of the ML estimate vs. the KR estimate. As in Figure 2, the ML estimate is less variable than the KR estimate. Since the true value of $\beta_{1}$ is known here, the mean squared error (MSE) of each estimator can be estimated as

$$
\begin{aligned}
\operatorname{MSE}\left(\hat{\beta}_{1}\right) & \approx 350^{-1} \sum_{i=1}^{350}\left[\left(\hat{\beta}_{1}\right)_{i}-\beta_{1}\right]^{2} \\
\operatorname{MSE}\left(\hat{\beta}_{1, \mathrm{ML}}\right) & \approx 0.015 \\
\operatorname{MSE}\left(\hat{\beta}_{1, \mathrm{KR}}\right) & \approx 0.021
\end{aligned}
$$


Table 3. Average lengths of KR and ML nominal 95\% confidence intervals and the percentage that cover $\beta_{1}=$ -0.3737 for the simulation study of Section 5 . The KR intervals have good coverage whereas the ML intervals do not

\begin{tabular}{lcc}
\hline Method & Average length & Per cent coverage \\
\hline Maximum likelihood & 0.168 & 44.9 \\
Krige and regress with $\widehat{\operatorname{var}}\left(\hat{\beta}_{1, \mathrm{KR}}\right)$ & 0.743 & 96.6 \\
Krige and regress with $\widehat{\operatorname{var}}_{\text {naïve }}$ & 0.667 & 96.9 \\
\hline
\end{tabular}

Both estimators are approximately unbiased. The mean of the ML estimates is -0.3661 , and the mean of the KR estimates is -0.3782 , whereas the true value of $\beta_{1}$ is -0.3737 .

Table 3 lists coverage of nominal KR 95\% confidence intervals where the standard error is calculated

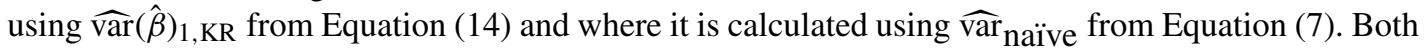
variance estimates give over $96 \%$ coverage. However, it is not recommended that the naive variance estimator be used in general because it ignores errors in the $\hat{\mathbf{X}}$ vector.

Table 3 also shows nominal ML 95\% confidence intervals where the standard error is calculated using the information-based asymptotic variance estimate $\widehat{\operatorname{var}}\left(\hat{\beta}_{1, \mathrm{ML}}\right)$ from Equation (17). The intervals cover $\beta_{1}$ only $44.9 \%$ of the time. In light of Figure 3 and Equation (21), the poor coverage must be due to the inadequacy of the asymptotic variance estimator.

\section{CONCLUSIONS}

The simulation study discussed in Section 5 confirms the results suggested in Section 4. The KR estimator of the slope parameter $\beta_{1}$ has a higher variance than the ML estimator does. The MSE of the KR estimator appears to be about $50 \%$ more than that of the ML estimator (0.015 vs. 0.021). However, the information-based asymptotic variance estimator for the ML estimator is much too small, giving only about $45 \%$ coverage with nominal $95 \%$ confidence intervals. A possible explanation for this is that the sample actually consists of only one (multivariate) observation, not a large enough sample for the asymptotics to be useful.

On the other hand, the KR variance estimator is based on the true finite-sample variance and performs fairly well in simulations, yielding over $96 \%$ coverage for $95 \%$ nominal confidence intervals. The naïve $\mathrm{KR}$ variance estimator, found by substituting $\hat{\mathbf{X}}$ in the standard WLS variance estimate formula, also performs well, but it is not clear that this will be true in general.

\section{ACKNOWLEDGEMENTS}

The research described in this paper has been partially funded by the U.S. Environmental Protection Agency through Cooperative Agreement CR-83168201-0: Program for Cooperative Research on Aquatic Indicators at Oregon State University. It has not been subjected to the Agency's review and therefore does not necessarily reflect the views of the Agency, and no official endorsement should be inferred.

\section{REFERENCES}

Cressie NAC. 1993. Statistics for Spatial Data (revised edn). John Wiley \& Sons, New York.

Gotway CA, Young LJ. 2002. Combining incompatible spatial data. Journal of the American Statistical Association 97: 632-648. 
Herlihy AT, Stoddard JT, Johnson CB. 1998. The relationship between stream chemistry and watershed land cover data in the Mid-Atlantic region, U.S. Water, Air, and Soil Pollution 105: 377-386.

Lehmann EL. 1998. Elements of Large-Sample Theory. Springer, New York.

Zhu L, Carlin B, Gelfand A. 2003. Hierarchical regression with misaligned spatial data: relating ambient ozone and pediatric asthma ER visits in Atlanta. Environmetrics 14: 537-557. 